\title{
Study on Effect of Grousers Mounted Flexible Wheel for Mobile Rovers
}

\author{
Kojiro Iizuka ${ }^{1}$ and Takashi Kubota ${ }^{2}$ \\ ${ }^{l}$ International Young Researchers Empowerment Center, Shinshu University, iizuka@shinshu-u.ac.jp \\ ${ }^{2}$ Japan Aerospace Exploration Agency, kubota@jaxa.isas.jp
}

\begin{abstract}
This paper presents the effectiveness of the flexible wheels for lunar exploration rovers. The flexible wheels give high performance while traversing loose soil. The flexible wheel is an effective scheme to traverse loose soil without poor performance. The flexible wheel has a surface which can be changed flexibly for rough terrain. When the surface of wheel performs matching the shape of ground it is crossing, the normal stress between the wheel and loose soil becomes small. It is prevented from sinking because this normal stress is small. However, the flexible wheel doesn't have high performance without grousers. The grouser is very important to get thrust for rovers. From running experiments using a single wheel tester, we found that the flexible wheel with grousers gave a high performance. The mounted positions of grousers on the surface of the wheel are defined by the same angle (ex) $36 \mathrm{deg}$ ). This means that there are two patterns to mount grousers. One is under the rib while the other is between the ribs. The spring force under the rib is different from between the ribs, it is greater. Moreover, each distortion is different. From these considerations, we need to find the optimal position for grousers. We carried out running experiments using wheels with both patterns. From our experiments, we understood the wheel with the position mounted between ribs gave a better performance than when it was set under the rib. When the area giving normal stress is wide, soil under the wheel becomes hard. Therefore, the wheel with grousers mounted between ribs gives a higher performance compared with the one mounted under the ribs.
\end{abstract}

\section{Keywords}

lunar rovers, flexible wheel, grousers, flexure, loose soil

\section{INTRODUCTION}

Robots are one of the most important mission devices for planetary exploration. They are designed to move on planetary surfaces for the purpose of collecting precise information regarding the origin of the solar system etc. The NASA Mars mission in 1997 was accomplished using the micro robot Sojourner that moved about and explored the surface of Mars. Sojourner transmitted important data and detailed images of the Martian surface to the Earth [NASA/JPL, Mars Path finder]. The Sojourner mission demonstrated the importance of mobile exploration. In planetary exploration robots are required to traverse rough terrains such as those found in craters and cliffs, scientifically important locations for exploration. Further, when moving over such terrain robots must avoid tipping over and getting stuck. Therefore, in order to improve the running performance of the wheels, we have used flexible material on the surface of the wheels. Figure 1(a) shows an ExoMars rover with wheels whose surface is made from flexible material developed by ESA [European Space Agency, ExoMars]. When the surface of the wheel is made from such flexible material, the contact area of the wheel with the ground becomes larger. Figure 1(b) shows a lunar rover with a wheel

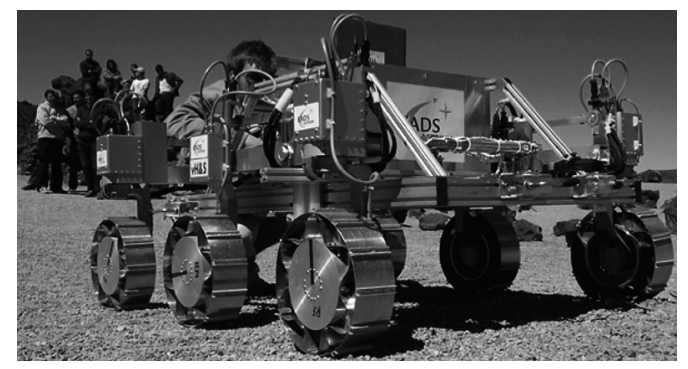

(a) ExoMars (ESA) [European Space Agency, ExoMars]

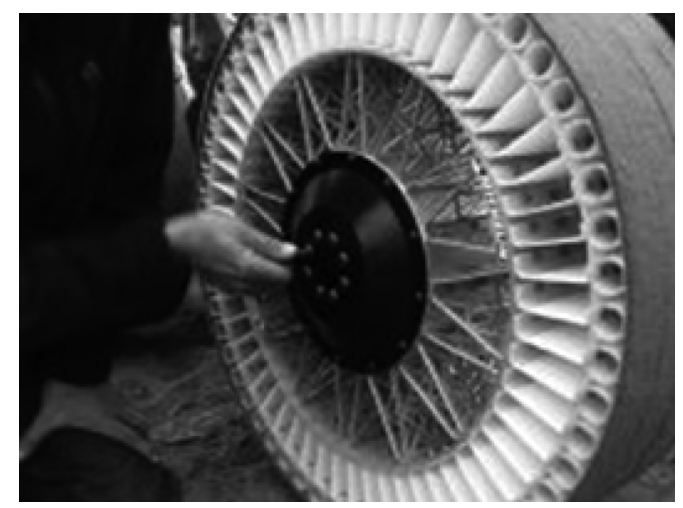

(b) Twheel (NASA/Mishilen) [Michelin Group]

Fig. 1 Rovers with the flexible wheels 
developed by NASA and Michelin [Michelin Group]. The form of the wheels of these rovers changes when they are traversing terrain; subsequently, the torque increases. The flexible wheel is configured on various parts: hub connected to motor; the outer circle; limbs connected between hub and outer circle. The limbs have an elastic property. When the flexible wheel rotates, the flexures of various positions are different. The flexures under the limbs are hard compared with flexures between the limbs.

We researched the effective positions of grousers using the flexible wheel with various rotational flexures. In experiments, the slip ratios of the flexible wheels with various setting positions of grousers were measured. From the results, we will look at the relationship between the position of grousers and the limbs of the flexible wheel.

In section 2, the flexible wheel proposed in this study is presented. In section 3 , the interaction between flexible wheels and loose soil is modeled. The experiments are presented in section 4. Its results and conclusions are described in section 5 .

Finally, the conclusions of the study are provided in Section 6.

\section{PROPOSED FLEXIBLE WHEEL}

The flexible wheel proposed for use in experiments in this study is shown in Figure 2. The proposed wheel is made of an elastic metallic material. The material used is copper beryllium. The Young's modulus and bending stress of copper beryllium are superior to those of other copper spring materials. The diameter of the wheel is $\varphi 200 \mathrm{~mm}$ and the width is $100 \mathrm{~mm}$. The size of the proposed wheel compares with that of the circular wheel.

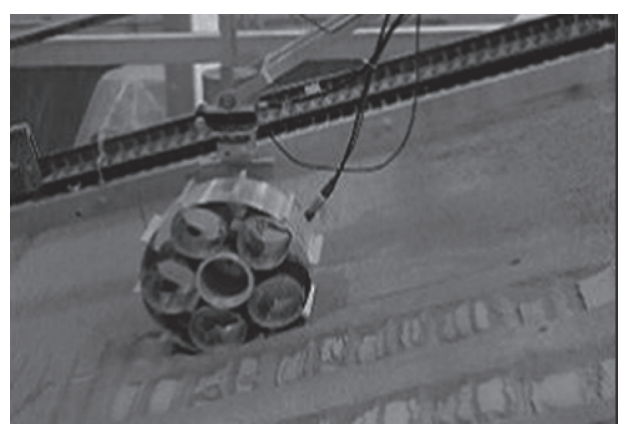

Fig. 2 Overview of running condition of proposed flexible wheel

\section{INTERACTION MODEL BETWEEN FLEX- IBLE WHEEL AND LOOSE SOIL}

Firstly, we will discuss the interaction model between a flexible wheel and loose soil. Over the past few years, several studies have been conducted using an interaction model with a circular solid wheel [Bekker, 1955; Yon et al.,1986; Yoshida et al., 2004; Iagnemma et al., 2006]. Muro modeled the interaction between low-stress tires and the ground [Muro, 1993]. Moreover, Ono considered FEM analysis using an elastic wheel [Ono, et al., 2010]. Size of the sand included a limit, but this was a very good method. Further, S.Narita studied a model using a spring [Narita et al., 2010]. The material of the wheels that are to be used on the lunar surface must be metal because of the unique environment on the lunar surface which includes vacuum conditions, radioactive rays, and large variations in temperature. A flexible wheel made of metal can change form. The material used on the surface of a flexible wheel is spring steel. When a wheel made using spring steel is loaded with weight, its form changes such that the part of the wheel that is in contact with the ground becomes flat. On this paper, the interaction model between a flexible wheel and loose soil is described in Figure 3(a). The surface of the flexible wheel becomes flat on the ground surface. Traction force is an important factor during traversal of the ground. This paper proposes an advanced model. This model is based on that of Muro. However, Muro's model does not consider the effect of the grousers on the wheels. Therefore, in this study, we

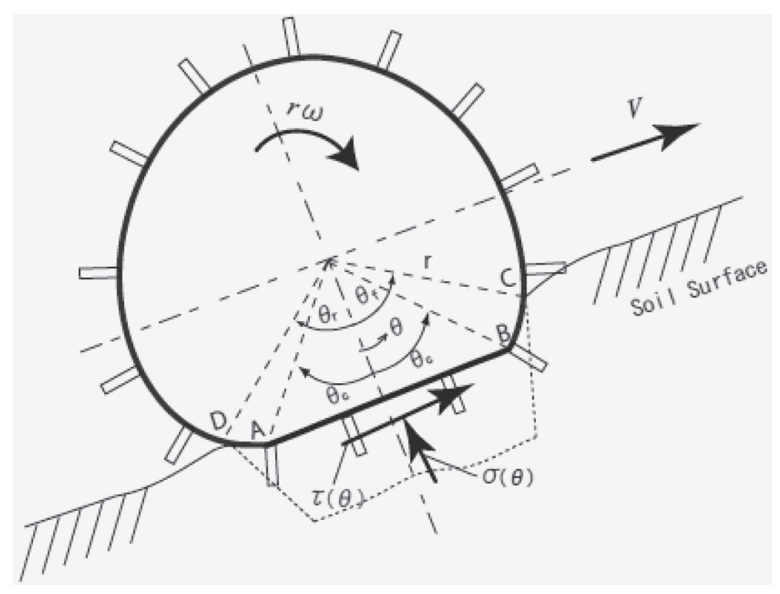

(a) Interaction model

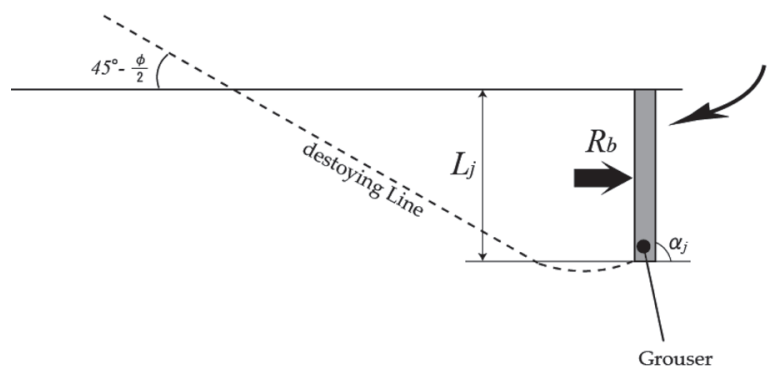

(b) Grouser model

Fig. 3 Interaction model between flexible wheel and loose soil 
propose an advanced model that includes the effect of the grousers. The traction force $D P$ is determined by various parameters such as the radius of the wheel $r$; the entry angle $\theta_{f}$; the exit angle $\theta_{c}$; the normal stress $\sigma(\theta)$; the shear stress $\tau(\theta)$; length of flat side between the points $A$ and $B, l_{A B}$; the reaction stress $R_{b}$; the length of the grousers $L_{j}$; the width of wheel, $b$; and the slip ratio, $\lambda$.

$$
\begin{aligned}
D P= & r b\left\{\int_{\theta_{c}}^{\theta_{1}} A d \theta+\frac{1}{r} \int_{0}^{l_{A B}} \tau(\theta) d x+\int_{\theta_{2}}^{\theta_{c}} A d \theta\right\} \\
& +L_{j} b \lambda\left\{\int_{\theta_{c}}^{\theta_{1}} B d \theta+\int_{0}^{l_{A B}} B \frac{1}{\cos \theta} d x+\int_{\theta_{2}}^{\theta_{c}} B d \theta\right\}
\end{aligned}
$$

where

$A=\tau(\theta) \cos \theta-\sigma(\theta) \sin \theta$

$B=R_{b} \cos \theta$

Interaction between the surface of the wheel and loose soil is shown in term 1 of equation (1). "From 0 to $l_{A B}$ " is expressed as a straight line on part of the flat condition of the elastic wheel. On the other, the effect by grousers is shown in term 2 of same equation (1). "From 0 to $l_{A B}$ " expressed as a straight line on part of the flat condition of the elastic wheel as same as in term 1. Moreover, term 2 of this model mean that the traction force increases when the slip ratio $\lambda$ increases. Further, the shearing stress $\tau(\theta)$ is expressed as

$\tau_{x}(\theta)=(c+\sigma(\theta) \tan \varphi)^{\{1-e-j x(\theta) / k x\}}$

The values of $c$ and $\varphi$ in equation (2) depend on the soil characteristics and the wheel surface. $\varphi$ is the friction angle of the soil. Figure 3(b) shows the grouser's model. When the grousers are inserted into the soil, the grousers can get the reaction stress from the soil. The reaction stress is expressed using the grouser length $L_{j}$ as

$R b=\frac{\sin \left(\alpha_{j}+\phi\right)}{\sin \alpha_{j}} C$

where

$C=L_{j} c\left(N_{c}-\tan \phi\right)+\frac{1}{2} \gamma L_{j}^{2}\left(\frac{2 N_{r}}{\tan \phi}+1\right)$

where

$\alpha_{j}$ : angle of approach

$\gamma$ : soil density

$L_{j}$ : grouser's length

$c$ : cohesion stress

$N_{c}, N_{r}$ : coefficients of sup port force
In this model, as observed in Figure 3(a), it is important to consider the flat part of the wheel, i.e., the part between points A and B. In this flat part, the reaction force applied by the soil to the wheel and also the grousers is significant. The sinkage $\mathrm{Z}$ can be expressed as follows.

$Z=r\left(\cos \theta_{c}-\cos \theta_{f}\right)$

When the sinkage becomes large, the resistance to running is large. In case of a flexible wheel, the sinkage does not easily become large and hence, the wheel does not sink because the surface of contact between the flexible wheel and the soil is flat. The flexible wheels have various parts, hub outer circle and limbs. The elastic effects of the limbs of the proposed flexible wheel are larger than them of the positions that are not under limbs. Figure 4 shows two of the condition positioned grousers on the flexible wheel. We research these conditions between the flexible wheels and the grousers.

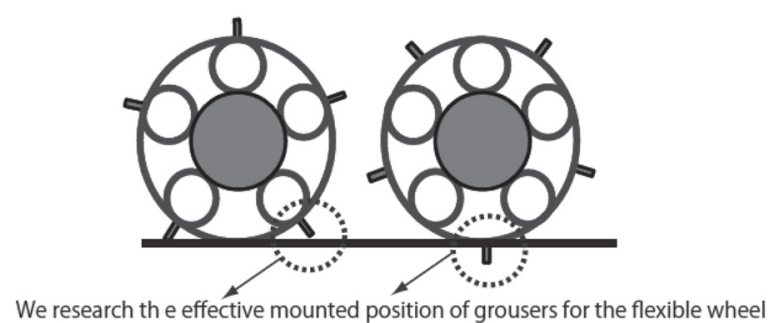

Fig. 4 Mounted position of grousers for flexible wheels

\section{EXPERIMENTS}

We measured two experiments. In experiment 1 , we measured the flexure value of the flexible wheel. In experiment 2, we measured the traversability of a proposed flexible wheel with various mounted cases of grousers by running a number of tests. In this (experiment 2), we focused on two effects. Firstly, on the effect of grousers; we compared the flexible wheel with and without grousers. Secondly, we researched the optimal mounted position of grousers. In experiment 2, we dealt with the flexible wheel with grousers set under each limb and then set between limbs. Both experiments used the slip ratio as traversability. To get these effects we used the single wheel test bed (Section 4-1).

\subsection{Single wheel test bed}

The configuration of the single wheel test bed is shown in Figure 5 to measure the slip ratio. The experimental system comprised of some mechanical parts and sensors, as shown in Figure 6. One wheel, a paral- 


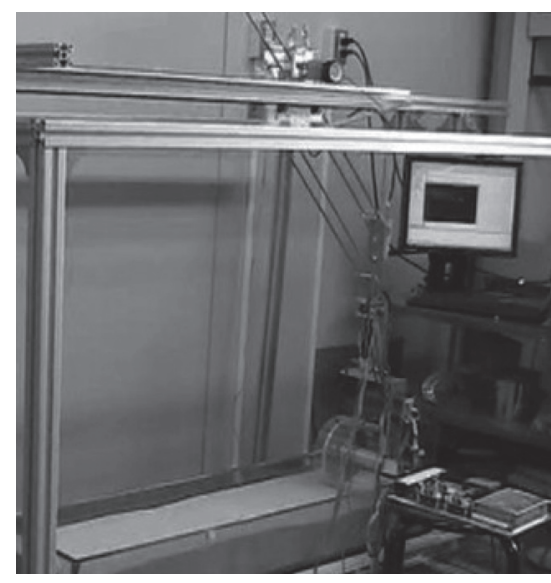

Fig. 5 View of experimental setup

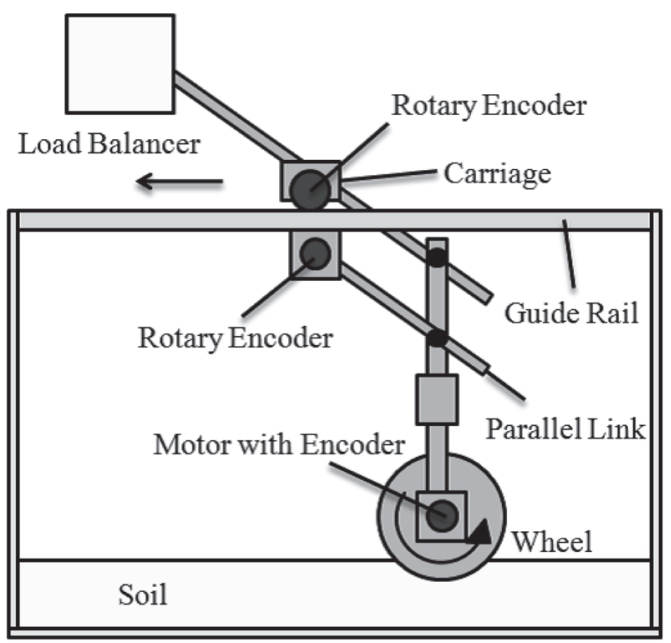

Fig. 6 Configuration of Experimental systems

lel link, stator, guide rail, load balance, and balance box were employed in the simulation. The parallel link was attached between the axis of the wheel and the load balance. The load balance ran on the guide rail. Sensors included a differential transformer and two encoders. The differential transformer measured the horizontal position of the wheel. The maximum distance measurable by the differential transformer is $1000 \mathrm{~mm}$. The vertical position of the wheel was calculated by a rotary encoder. The velocity of the wheel was calculated by using the vertical and horizontal positions and the time taken by the wheel to cover a certain distance. The rotation of the wheel was obtained from the encoder. The slip ratio was calculated using the values of velocity and rotation. The sinkage determined by measuring the depth of the soil before and after running the experiments, thereby estimating the difference. The load of wheel was set by placing the appropriate weight on the balance box. This study uses the slip ratio as the evaluation of performance of the wheels. The slip ratio is the ratio between the angular velocity of wheel and the actual displacement moved by wheel. The equation of slip ratio $\lambda$ is followed using velocity $V$, radius of the wheel $r$ and angular velocity of the wheel $\omega$;

$$
\lambda=1-\frac{v}{r \omega}(6)
$$

\subsection{Experiment 1: Measurements on the flexibility of the proposed wheel}

We measured the flexure value of the flexible wheel. The two positions of the flexible wheel were measured as shown in Figure 7 (flexure under limbs and then between limbs). In measurements, we used the weights from 0 to $50 \mathrm{~N}$ to load the flexible wheel.
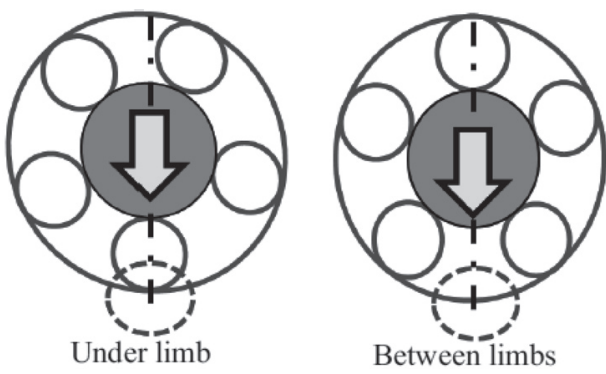

Fig. 7 Position to measure flexure of flexible wheel

\subsection{Experiment 2: Traversability (Level of slip)}

In the parameters experiment, the wheel load, speed of the wheel and slope angle could be varied. The experimental parameters are shown in Table 1. The patterns of the wheels used in the experiments are shown in Figure 8. Firstly, we carried out the running experiments using a proposed flexible wheel with and without grousers. Secondly, we focused on the mounted position of the grousers. In the experiments, we used four wheels as follows:

(a) Without grousers

(b) Grousers mounted under limbs

Table 1 Experimental parameters

\begin{tabular}{|l|l|}
\hline Load & $3.0 \mathrm{~kg}$ \\
\hline Speed & $10 \mathrm{~mm} / \mathrm{s}$ \\
\hline Slope & $0,5,10,15,20^{\circ}$ \\
\hline Soil used & Keisa No.5 (JIS Standard soil) \\
\hline
\end{tabular}

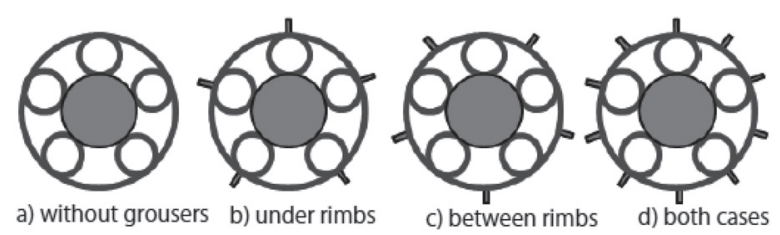

Fig. 8 Experimental wheels 
(c) Grousers set between limbs

(d) With grousers mounted both under and between the limbs

From these experiments, we obtained knowledge of the effect of grousers and their effective mounted positions.

\section{RESULTS AND DISCUSSION}

\subsection{Flexure of flexible wheel}

Figure 9 is the view of the flexure of the proposed wheel between limbs. Figure 10 shows the view of the flexure of this wheel under the limbs. When each wheel has the same weight we discovered that each flexure was different. Figure 11 shows the measurement values of flexure of the flexible wheel. When the weight of the flexible wheel increases, the values of

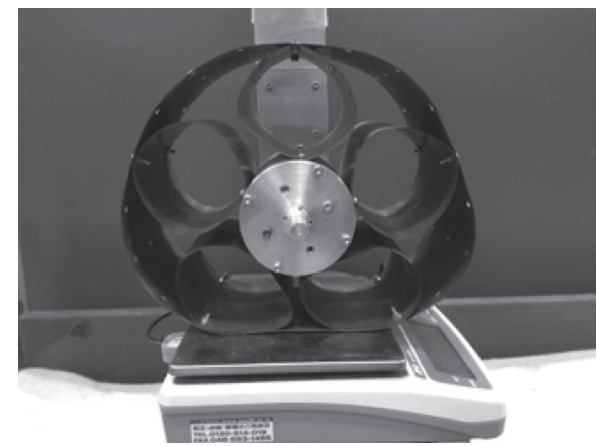

Fig. 9 Flexure of flexible wheel (between the limbs)

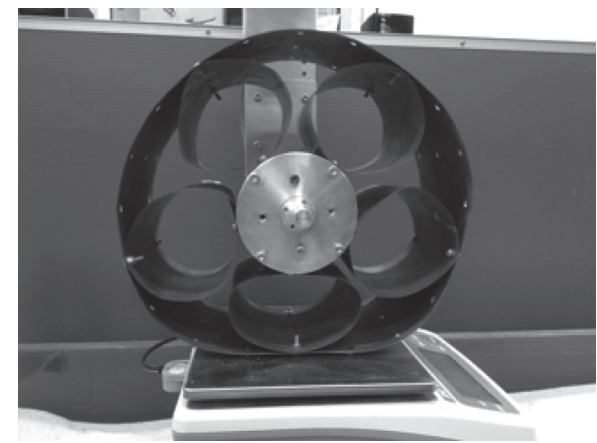

Fig. 10 Flexure of flexible wheel (under limb)

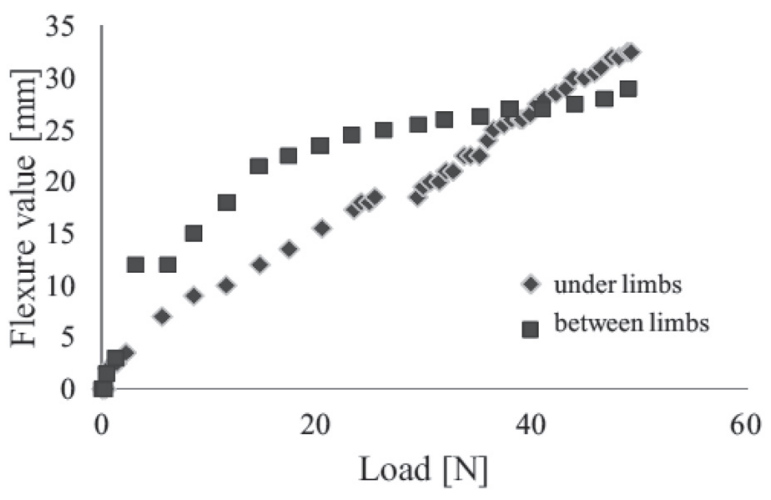

Fig. 11 Flexure value of flexible wheel flexure became larger. Moreover, there was a difference of flexure between "under limbs" and "between limbs". Until $40 \mathrm{~N}$, the flexure under limbs was constant. On the other hand, the flexure between limbs increases rapidly until $20 \mathrm{~N}$. Then we discovered that the flexure of this was not easy to increase over 20 $\mathrm{N}$. When both wheels were compared, the flexure between limbs was larger than that under limbs until 40 $\mathrm{N}$. When the loaded weight increased over $40 \mathrm{~N}$, the flexure value under limbs became larger than that between limbs.

\subsection{Slip ratio of the flexible wheels with various po- sitions of grousers}

The running view of the proposed flexible wheel without grousers is shown in Figure 12. When the slope for running becomes steeper, the one without grousers slips easily. Figure 13 shows the running view of the one with grousers mounted under limbs. Figure 14 shows the running view of the proposed wheel with grousers mounted between limbs. The proposed flexible wheel with grousers mounted between the limbs slips more compared to the one mounted under limbs. Figure 15 shows the running view of the one with grousers mounted both under and between the limbs. The slip behavior of this wheel is low for all wheels. We believe that the numbers of grousers are very

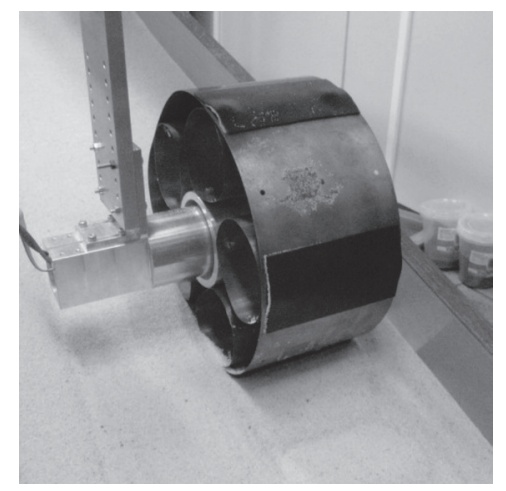

Fig. 12 Running view of wheel without grousers

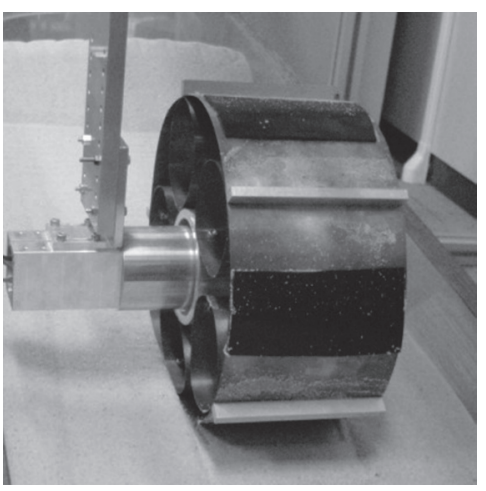

Fig. 13 Running view of wheel with grousers under limbs 


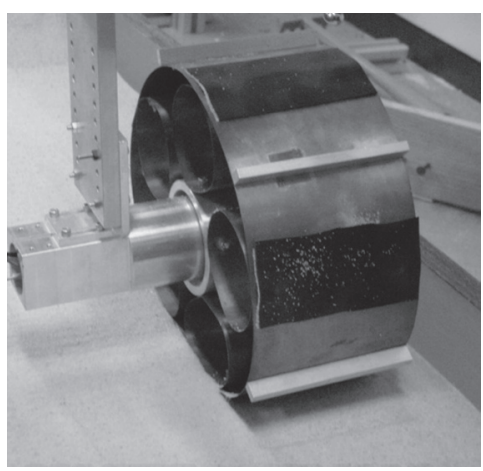

Fig. 14 Running view of wheel with grousers between limbs

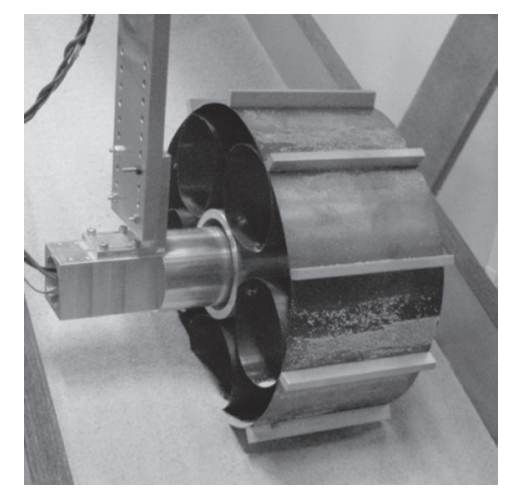

Fig. 15 Running view of wheel with grousers under and between

important. Figure 16 shows the slip ratio of the flexible wheel without grousers. When the slope becomes steep, the slip ratio becomes higher. The slip ratio became around $0.7-0.8$ at $20 \mathrm{deg}$. Figure 17 shows the slip ratio of the flexible wheels with grousers (10 grousers each $36 \mathrm{deg}$ ). The slip ratio of that is smaller than that without grousers. When this flexible wheel traverses loose soil at $20 \mathrm{deg}$ the slip ratio is around $0.2-0.3$. This value is very small, and it means that the flexible wheel with grousers has a high performance. Figure 18 shows the slip ratio of the flexible wheel

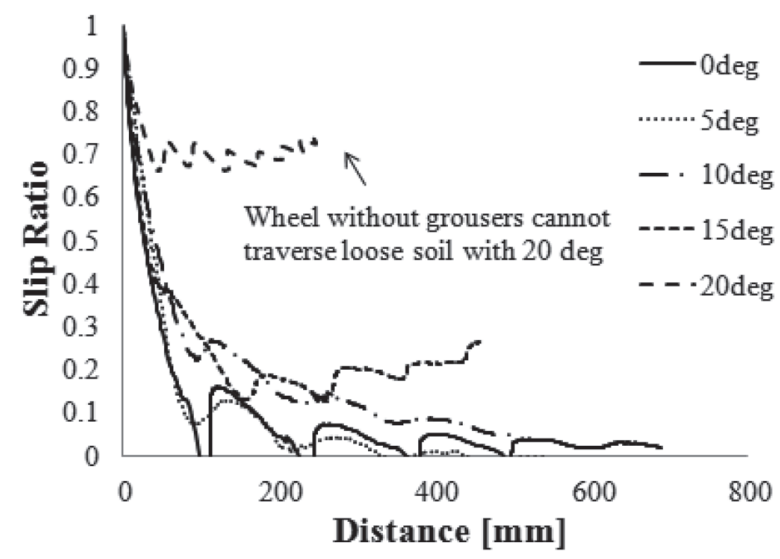

Fig. 16 Result: without grousers

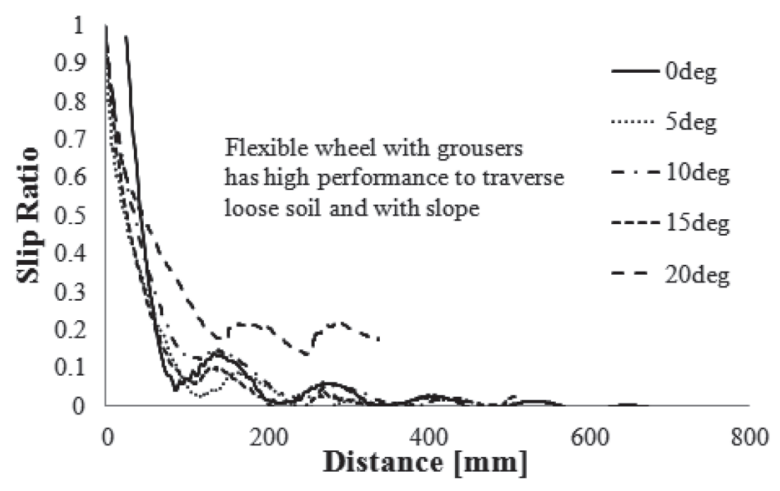

Fig. 17 Result: with grousers

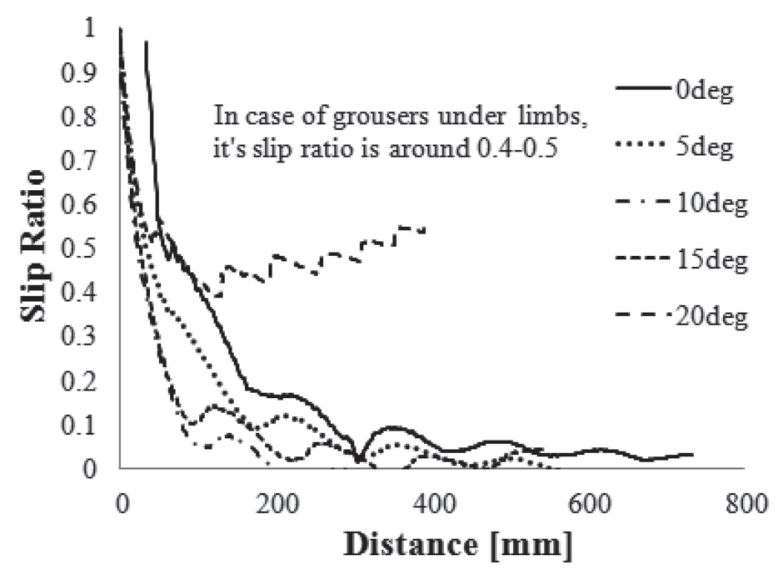

Fig. 18 Result: Slip ratio of flexible wheel with grousers under limbs

with grousers positioned under limbs. When the slope increases, the slip ratio becomes higher. At $20 \mathrm{deg}$ the slip ratio is around 0.5-0.7.

Figure 19 shows the slip ratio of the flexible wheel with grousers positioned between limbs. The slip ratio has a low value when the slope is low. However, the slip ratio at $20 \mathrm{deg}$ became a lot higher. When we compared the results of that under the limb to that between limbs we found that the slip ratio of the flexible wheel with grousers positioned between limbs had

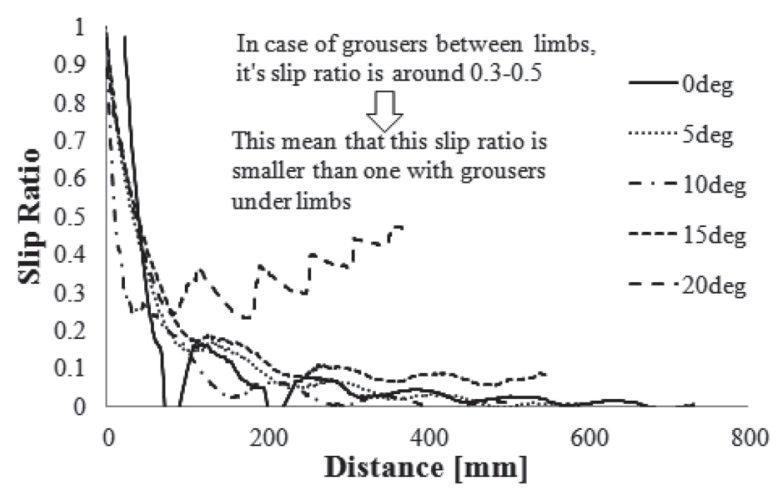

Fig. 19 Result: Slip ratio of flexible wheel with grousers between limbs 
a low value (0.3-0.5). From these results, it is effective to position grousers between limbs for traversing loose soil.

\section{CONCLUSION}

This study considered the effect of grousers for the flexible wheel. Especially, we focused on the position of grousers. From the experimental results, the flexible wheel with grousers has a high performance. Moreover, the running performance of the flexible wheel with grousers positioned between limbs is better than when they are positioned under the limbs. From these discussions we obtained knowledge of the most effective position for grousers on a flexible wheel.

\section{Acknowledgements}

This study was partly supported by Program for Dissemination of Tenure-Track System funded by the Ministry of Education and Science, Japan.

\section{References}

Bekker, M., Theory of Land Locomotion, The University of Michigan Press, 1955.

European Space Agency, http://www.esa.int/esaMI/ ExoMars/index.html.

Mars Pathfinder, http://mars.jpl.nasa.gov/MPF/.

Michelin Group, http://www.michelin.com/.

Iagnemma, K., S. Kang, H. Shibly, and S. Dubowsky, Online terrain parameter estimation for wheeled mobile robots with application to planetary robots, IEEE Transactions on Robotics, Vol. 20, No. 5, 921-927, 2004.

Ishigami, G., K. Nagatani, and K. Yoshida, Path following control with slip compensation on loose soil for exploration rover, Proceedings of 2006 IEEE/ RSJ International Conference on Intelligent Robots and Systems, 2006.

Muro, T., Terramechanics: Running Dynamics, Gihodo Press, 1993.

Narita, S., M. Otuki, S. Wakabayashi, and S. Nishida, Mobility evaluation of low-pressure wheel lunar exploration rover, Proceedings of the Joint 9th Asia-Pacific ISTVS Conference and Annual Meeting of Japanese Society for Terramechanics, No. 085, DVD-Rom, 2010.

Ono, T., H. Nakashima, H. Shimizu, J. Miyasaka, K. Ohdoi, FE-DEM analysis of tractive performance of an elastic wheel for planetary rover, Proceedings of the Joint 9th Asia-Pacific ISTVS Conference and Annual Meeting of Japanese Society for Terramechanics, No. 082, DVD-Rom, 2010.

Yon, R. N., E. A. Fattah, N. Skidas, and M. Kitano, Run dynamics of off-road vehicles, The Japanese Society for Study Technology Education, 1986
Yoshida, K., N. Mizuno, G. Ishigami, and A. Miwa, Terramechanics-based analysis for slope climbing capability of a lunar/planetary rover, Proceedings of 24th International Symposium on Space Technology and Science, 2004-k-06, 2004.

(Received April 4, 2012; accepted May 14, 2012) 